\title{
Correlated imaging, quantum and classical
}

\author{
A. Gatti, E. Brambilla, M. Bache, and L. A. Lugiato \\ INFM, Dipartimento di Scienze CC.FF.MM., Università dell'Insubria, Via Valleggio 11, 22100 Como, Italy
}

(Received 9 December 2003; published 6 July 2004)

\begin{abstract}
We analytically show that it is possible to perform coherent imaging by using the classical correlation of two beams obtained by splitting incoherent thermal radiation. A formal analogy is demonstrated between two such classically correlated beams and two entangled beams produced by parametric down-conversion. Because of this analogy, the classical beams can mimic qualitatively all the imaging properties of the entangled beams, even in ways which up to now were not believed possible. A key feature is that these classical beams are spatially correlated both in the near field and in the far field. Using realistic numerical simulations the performances of a quasithermal and a parametric down-conversion source are shown to be closely similar, both for what concerns the resolution and statistical properties. The results of this paper provide a scenario for the discussion of what role the entanglement plays in correlated imaging.
\end{abstract}

DOI: 10.1103/PhysRevA.70.013802

PACS number(s): 42.50.Dv, 42.50.Ar

\section{INTRODUCTION}

The topic of entangled imaging has attracted noteworthy attention in recent years [1-9]. This technique exploits the quantum entanglement of the state generated by parametric down-conversion (PDC), in order to retrieve information about an unknown object. In the regime of single photon-pair production of PDC, the photons of a pair are spatially separated and each propagates through a distinct imaging system, usually called the test and the reference arm. An object is located in the test arm. Information about the spatial distribution of the object is obtained by registering the coincidence counts as a function of the transverse position of the photon in the reference arm, which holds a known reference system [1-6]. In the regime of a large number of photon pairs, this procedure is generalized to the measurement of the signal-idler spatial correlation function of intensity fluctuations [7]. Such a two-arm configuration provides more flexibility in comparison with standard imaging procedures. For example, there is the possibility of illuminating the object at a given light frequency in the test arm and of performing a spatially resolved detection in the other arm with a different light frequency, or of processing the information from the object by only operating on the imaging system of the reference arm [6]. In addition, it opens the possibility for performing coherent imaging by using, in a sense, spatially incoherent light, since each of the two down-converted beams taken separately is described by a thermallike mixture and only the two-beam state is pure (see Refs. [5,7]).

In this paper (see also Ref. [29]) we show that such a scheme can be implemented using truly incoherent light, as the radiation produced by a thermal (or quasithermal) source. A comparison between thermal and photon-pair emission was performed in Ref. [10], where an underlying duality accompanies the mathematical similarity between the two cases. Here we consider a different scheme (Fig. 1) appropriate for correlated imaging, in which a thermal beam is divided by a beam splitter (BS) and the two outgoing beams are handled in the same way as the PDC beams in entangled imaging. Our analysis points out a precise formal analogy between the PDC and the thermal case. This analogy opens the possibility for using classically correlated thermal light for correlated imaging in the same way as entangled beams from PDC.

Currently there is a very lively debate whether quantum entanglement is a necessary ingredient to perform correlated imaging [5,7-9]. The first coincidence imaging experiments were performed using entangled photons from PDC [3,4]. At that time, the authors of Ref. [3] suggested that "it is possible to imagine some type of classical source that could partially emulate this behavior." A more recent theoretical analysis [5] gave arguments that "the distributed quantum-imaging scheme truly requires entanglement in the source and cannot be achieved using a classical source with correlations but without entanglement." The topic became hot after the ghost image experiment of Ref. [3] was successfully reproduced using classically correlated beams [8]. In this experiment a classical source produced pairs of single-mode angularly correlated pulses that served as classical analogs of momentum correlated pairs of photons produced by PDC. In the accompanying theoretical discussion, the authors presented argu-

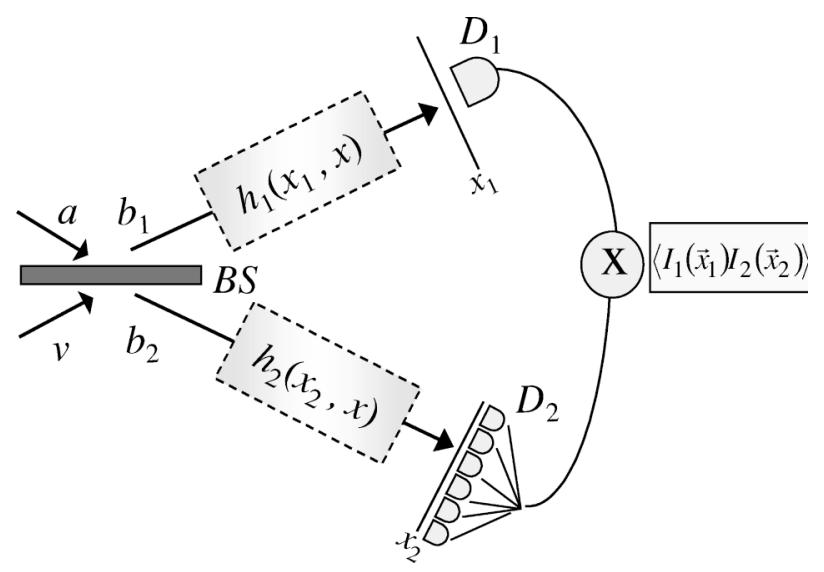

FIG. 1. Correlated imaging with incoherent thermal light. The thermal beam $a$ at the beam splitter BS is divided into two beams, $b_{1}$ and $b_{2}$, which travel through, respectively, a test and a reference system, described by their impulse response functions $h_{1}$ and $h_{2}$. The test arm 1 includes an object. Detector $D_{1}$ is either a pointlike detector or a bucket detector. $D_{2}$ is an array of pixel detectors. $v$ is a vacuum field. 
ments that although the results of any single experiment in quantum imaging could be reproduced by classical sources with proper statistical correlation, a given classical source cannot emulate the behavior of a quantum entangled source for any arbitrary test and reference systems. In agreement with this we showed in a recent theoretical paper [7] that on the one hand the results of any single quantum-imaging experiment performed with entangled beams in a pure state could be exactly reproduced by using separable (i.e., nonentangled) states. On the other hand, we showed that a key feature of the entangled state produced by PDC is the simultaneous presence of spatial correlation at the quantum level in the near field and the far field of the signal-idler beams (see also Ref. [11]). This corresponds to the simultaneous quantum correlation of position and momentum of the photons in each pair. We showed that this feature could be exploited to produce both the image and the diffraction pattern of an object by solely operating on the reference arm, and argued that this could be done only in the presence of quantum entanglement. This interpretation was received rather well in the quantum-imaging community and was generally viewed as a possibility to discriminate between the presence of quantum entanglement and classical correlation in the source.

In this paper we will analyze a counter example, which partially contradicts the picture emerging from Refs. [7,8]. The classical, thermal beams created by splitting thermal radiation have several features that distinguish them from other nonentangled beams considered in the previous literature $[7,8]$. First of all, they are spatially multimode, unlike those considered in Ref. [8]. Second, as we will see in Sec.IV they have an imperfect level of spatial correlation, limited by the classical shot noise introduced by the beam splitter. Nonetheless, they exhibit a high level of spatial correlation in both the near-field and far-field planes, in contrast to the separable mixtures considered in Ref. [7] that were perfectly correlated in either plane. We will show that this imperfect spatial correlation in both planes, although being completely classical, is enough to qualitatively reproduce all the features of the entangled imaging, provided that the spatial coherence properties of the thermal source are properly engineered. Finally, they are probably the best classical analogs of the entangled signal-idler beams produced by PDC, since the marginal statistics of the signal or idler beam alone is a thermal statistics. Thus, they should provide an optimal test bed for understanding the role of entanglement in correlated imaging.

In Sec. II we demonstrate theoretically the analogy between thermal and entangled PDC beams in correlated imaging. Section III discusses in analytical terms a specific imaging scheme. In Sec. IV we discuss the origin of the spatial correlation in the thermal case and relate it to the entangled case. In Sec. V the performances of the two cases are compared and we show a key numerical example that drops the spatiotemporal translational invariance assumed in the analytical treatment. Section VI contains the conclusions and a discussion. Finally, the Appendix discusses the degree and visibility of the correlation between the thermal beams when the finite detection area of the measurement apparatus is taken into account.

\section{ANALOGY BETWEEN THERMAL AND ENTANGLED BEAMS IN CORRELATED IMAGING}

In this section we are going to show a close analogy between the use of thermal light and entangled beams from PDC in the imaging schemes based on correlation measurements. For the sake of comparison, the two cases will be treated in parallel. In the analytical treatment we consider for simplicity only spatial variables and ignore the time argument, which corresponds to using a narrow frequency filter. We will come back to this point in Sec. V. In addition, we assume translational invariance in the transverse plane, which amounts to requiring that the cross section of the source is much larger than the object and all the optical elements.

In the entangled case, the signal and idler fields are generated in a type II $\chi^{(2)}$ crystal by a PDC process. Our starting point is the input-output relations of the crystal, which in the plane-wave pump approximation read $[7,11,12]$

$$
b_{i}(\vec{q})=U_{i}(\vec{q}) a_{i}(\vec{q})+V_{i}(\vec{q}) a_{j}^{\dagger}(-\vec{q}), \quad i \neq j=1,2 .
$$

Here, $b_{i}(\vec{q})=\int(\mathrm{d} \vec{x} / 2 \pi) e^{-i \vec{q} \cdot \vec{x}} b_{i}(\vec{x})$, where $b_{i}(\vec{x})$ are the signal $(i=1)$ and idler $(i=2)$ field envelope operators at the output face of the crystal (distinguished by their orthogonal polarizations), $\vec{x}$ being the position in the transverse plane. $a_{i}, i$ $=1,2$ are the corresponding fields at the input face of the crystal, and are taken to be in the vacuum state. The gain functions $U_{i}, V_{i}$ are for example given in Ref. [11].

In the thermal case, we start from the input-output relations of a beam splitter

$$
b_{1}(\vec{x})=r a(\vec{x})+t v(\vec{x}), \quad b_{2}(\vec{x})=t a(\vec{x})+r v(\vec{x}),
$$

where $t$ and $r$ are the complex transmission and reflection coefficients of the mirror, $a$ is a thermal field, and $v$ is a vacuum field uncorrelated from $a$. We assume that the thermal state $a(\vec{x})$ is characterized by a Gaussian field statistics, in which any correlation function of arbitrary order is expressed via the second-order correlation function [13]:

$$
\Gamma\left(\vec{x}, \vec{x}^{\prime}\right)=\left\langle a^{\dagger}(\vec{x}) a\left(\vec{x}^{\prime}\right)\right\rangle=\int \frac{d \vec{q}}{(2 \pi)^{2}} e^{-i \vec{q}\left(\vec{x}-\vec{x}^{\prime}\right)}\langle n(\vec{q})\rangle_{\text {th }} .
$$

Here $\langle n(\vec{q})\rangle_{\text {th }}$ denotes the expectation value of the photon number in mode $\vec{q}$ in the thermal state. In writing the second line of this equation, we implicitly used the hypothesis of translational invariance of the source, under which $\Gamma\left(\vec{x}, \vec{x}^{\prime}\right)$ $=\Gamma\left(\vec{x}-\vec{x}^{\prime}\right)$. In particular, the following factorization property holds [13]:

$$
\begin{aligned}
\left\langle: a^{\dagger}(\vec{x}) a\left(\vec{x}^{\prime}\right) a^{\dagger}\left(\vec{x}^{\prime \prime}\right) a\left(\vec{x}^{\prime \prime \prime}\right):\right\rangle= & \left\langle a^{\dagger}(\vec{x}) a\left(\vec{x}^{\prime}\right)\right\rangle\left\langle a^{\dagger}\left(\vec{x}^{\prime \prime}\right) a\left(\vec{x}^{\prime \prime \prime}\right)\right\rangle \\
& +\left\langle a^{\dagger}(\vec{x}) a\left(\vec{x}^{\prime \prime \prime}\right)\right\rangle\left\langle a^{\dagger}\left(\vec{x}^{\prime \prime}\right) a\left(\vec{x}^{\prime}\right)\right\rangle,
\end{aligned}
$$

where :: indicates normal ordering.

In both the PDC and the thermal case each of the two outgoing beams travels through a distinct imaging system, described by its impulse response functions $h_{1}\left(\vec{x}_{1}, \vec{x}_{1}^{\prime}\right)$ and $h_{2}\left(\vec{x}_{2}, \vec{x}_{1}^{\prime}\right)$, respectively (see Fig. 1). Arm 1 includes an object. Beam 1 is detected by $D_{1}$, which is either a pointlike detector 
or by a "bucket" detector which collects all the light in the detection plane [6]; in any case $D_{1}$ gives no information on the object spatial distribution. In the other arm the detector $D_{2}$ spatially resolves the light fluctuations, as for example an array of pixel detectors. The fields at the detection planes are given by

$$
c_{i}\left(\vec{x}_{i}\right)=\int d \vec{x}_{i}^{\prime} h_{i}\left(\vec{x}_{i}, \vec{x}_{i}^{\prime}\right) b_{i}\left(\vec{x}_{i}^{\prime}\right)+L_{i}\left(\vec{x}_{i}\right), \quad i=1,2,
$$

where $L_{1}, L_{2}$ account for possible losses in the imaging systems, and depend on vacuum field operators uncorrelated from $b_{1}, b_{2}$. Information about the object is extracted by measuring the spatial correlation function of the intensities detected by $D_{1}$ and $D_{2}$, as a function of the position $\vec{x}_{2}$ of the pixel of $D_{2}$ :

$$
\left\langle I_{1}\left(\vec{x}_{1}\right) I_{2}\left(\vec{x}_{2}\right)\right\rangle=\left\langle c_{1}^{\dagger}\left(\vec{x}_{1}\right) c_{1}\left(\vec{x}_{1}\right) c_{2}^{\dagger}\left(\vec{x}_{2}\right) c_{2}\left(\vec{x}_{2}\right)\right\rangle .
$$

All the object information is concentrated in the correlation function of intensity fluctuations:

$$
G\left(\vec{x}_{1}, \vec{x}_{2}\right)=\left\langle I_{1}\left(\vec{x}_{1}\right) I_{2}\left(\vec{x}_{2}\right)\right\rangle-\left\langle I_{1}\left(\vec{x}_{1}\right)\right\rangle\left\langle I_{2}\left(\vec{x}_{2}\right)\right\rangle,
$$

where $\left\langle I_{i}\left(\vec{x}_{i}\right)\right\rangle=\left\langle c_{i}^{\dagger}\left(\vec{x}_{i}\right) c_{i}\left(\vec{x}_{i}\right)\right\rangle$ is the mean intensity of the $i$ th beam. When using a bucket detector in arm 1, the measured quantity corresponds to the integral over $\vec{x}_{1}$ of both sides of Eq. (7). Since $c_{1}$ and $c_{2}^{\dagger}$ commute, all the terms in Eqs. (6) and (7) are normally ordered and $L_{1}, L_{2}$ can be neglected, thus obtaining

$$
\begin{aligned}
G\left(\vec{x}_{1}, \vec{x}_{2}\right)= & \int d \vec{x}_{1}^{\prime} \int d \vec{x}_{i}^{\prime \prime} \int d \vec{x}_{2}^{\prime} \int d \vec{x}_{2}^{\prime \prime} h_{1}^{*}\left(\vec{x}_{1}, \vec{x}_{1}^{\prime \prime}\right) h_{1}\left(\vec{x}_{1}, \vec{x}_{1}^{\prime}\right) h_{2}^{*}\left(\vec{x}_{2}, \vec{x}_{2}^{\prime \prime}\right) h_{2}\left(\vec{x}_{2}, \vec{x}_{2}^{\prime}\right) \\
& \times\left[\left\langle b_{1}^{\dagger}\left(\vec{x}_{1}^{\prime \prime}\right) b_{1}\left(\vec{x}_{1}^{\prime}\right) b_{2}^{\dagger}\left(\vec{x}_{2}^{\prime \prime}\right) b_{2}\left(\vec{x}_{2}^{\prime}\right)\right\rangle-\left\langle b_{1}^{\dagger}\left(\vec{x}_{1}^{\prime \prime}\right) b_{1}\left(\vec{x}_{1}^{\prime}\right)\right\rangle\left\langle b_{2}^{\dagger}\left(\vec{x}_{2}^{\prime \prime}\right) b_{2}\left(\vec{x}_{2}^{\prime}\right)\right\rangle\right] .
\end{aligned}
$$

In the thermal case, by taking into account the transformation (2) and that $v$ is in the vacuum state, $b_{1}$ and $b_{2}$ in Eq. (8) can be simply replaced by $r a$ and $t a$, respectively. Next, by using Eq. (4), we arrive at the final result

$$
\begin{aligned}
G_{\mathrm{th}}\left(\vec{x}_{1}, \vec{x}_{2}\right)= & |\operatorname{tr}|^{2} \mid \int d \vec{x}_{1}^{\prime} \int d \vec{x}_{2}^{\prime} h_{1}^{*}\left(\vec{x}_{1}, \vec{x}_{1}^{\prime}\right) h_{2}\left(\vec{x}_{2}, \vec{x}_{2}^{\prime}\right) \\
& \times\left.\left\langle a^{\dagger}\left(\vec{x}_{1}^{\prime}\right) a\left(\vec{x}_{2}^{\prime}\right)\right\rangle\right|^{2},
\end{aligned}
$$

where $\left\langle a^{\dagger}\left(\vec{x}_{1}^{\prime}\right) a\left(\vec{x}_{2}^{\prime}\right)\right\rangle$ is given by Eq. (3).

Similarly, the four-point correlation function in Eq. (8) has special factorization properties also in the PDC case. As it can be obtained from Eq. (1) (see also Ref. [11]),

$$
\begin{aligned}
\left\langle b_{1}^{\dagger}\left(\vec{x}_{1}^{\prime \prime}\right) b_{1}\left(\vec{x}_{1}^{\prime}\right) b_{2}^{\dagger}\left(\vec{x}_{2}^{\prime \prime}\right) b_{2}\left(\vec{x}_{2}^{\prime}\right)\right\rangle= & \left\langle b_{1}^{\dagger}\left(\vec{x}_{1}^{\prime \prime}\right) b_{1}\left(\vec{x}_{1}^{\prime}\right)\right\rangle\left\langle b_{2}^{\dagger}\left(\vec{x}_{2}^{\prime \prime}\right) b_{2}\left(\vec{x}_{2}^{\prime}\right)\right\rangle \\
& +\left\langle b_{1}^{\dagger}\left(\vec{x}_{1}^{\prime \prime}\right) b_{2}^{\dagger}\left(\vec{x}_{2}^{\prime \prime}\right)\right\rangle\left\langle b_{1}\left(\vec{x}_{2}^{\prime}\right) b_{2}\left(\vec{x}_{2}^{\prime}\right)\right\rangle .
\end{aligned}
$$

By inserting this result in Eq. (8), one obtains

$$
\begin{aligned}
G_{\mathrm{PDC}}\left(\vec{x}_{1}, \vec{x}_{2}\right)= & \mid \int d \vec{x}_{1}^{\prime} \int d \vec{x}_{2}^{\prime} h_{1}\left(\vec{x}_{1}, \vec{x}_{i}^{\prime}\right) h_{2}\left(\vec{x}_{2}, \vec{x}_{2}^{\prime}\right) \\
& \times\left.\left\langle b_{1}\left(\vec{x}_{1}^{\prime}\right) b_{2}\left(\vec{x}_{2}^{\prime}\right)\right\rangle\right|^{2},
\end{aligned}
$$

where by using relations (1)

$$
\left\langle b_{1}\left(\vec{x}_{1}^{\prime}\right) b_{2}\left(\vec{x}_{2}^{\prime}\right)\right\rangle=\int \frac{d \vec{q}}{(2 \pi)^{2}} e^{i \vec{q} \cdot\left(\vec{x}_{1}^{\prime}-\vec{x}_{2}^{\prime}\right)} U_{1}(\vec{q}) V_{2}(-\vec{q}) .
$$

At this point the analogy between the results in the two cases clearly emerges. Apart from the numerical factor $|t r|^{2}$ and the presence of $h_{1}^{*}$ instead of $h_{1}$, the thermal second-order correlation $\left\langle a^{\dagger}(\vec{x}) a\left(\vec{x}^{\prime}\right)\right\rangle$ in Eq. (9) plays the same role as the PDC signal-idler correlation function $\left\langle b_{1}(\vec{x}) b_{2}\left(\vec{x}^{\prime}\right)\right\rangle$ in Eq. (11). Consequently from Eqs. (3) and (12), the thermal mean photon number $\langle n(\vec{q})\rangle_{\text {th }}$ plays the same role as $U_{1}(\vec{q}) V_{2}(-\vec{q})$ in the PDC case. The correlation function $\left\langle a^{\dagger}(\vec{x}) a\left(\vec{x}^{\prime}\right)\right\rangle$ governs the properties of spatial coherence of the thermal source $[13,14]$. The correlation length, or transverse coherence length $l_{\text {coh }}$, is determined by the inverse of the bandwidth $\Delta q$ of the function $\langle n(\vec{q})\rangle_{\mathrm{th}}$. The same comments hold for the correlation $\left\langle b_{1}(\vec{x}) b_{2}\left(\vec{x}^{\prime}\right)\right\rangle$, and the function $U_{1}(\vec{q}) V_{2}(-\vec{q})$ in the entangled case. Most importantly, unlike the results for the separable states considered in Refs. [5,7], in both Eqs. (9) and (11) the modulus is outside the integral; this is the feature that ensures the possibility of coherent imaging via the correlation function (see, e.g., Ref. [5]).

\section{IMAGING SCHEMES: IMAGE AND DIFFRACTION PATTERN OF AN OBJECT}

Let us now analyze two paradigmatic examples of imaging systems, borrowed from the discussion of Ref. [7] and sketched in Fig. 2.

In both examples the setup of arm 1 is fixed, and consists of an object, described by a complex transmission function $T(\vec{x})$, and a lens located at a focal distance $f$ from the object and from the detection plane. Hence,

$$
h_{1}\left(\vec{x}_{1}, \vec{x}_{1}^{\prime}\right)=-\frac{i}{\lambda f} \exp \left(-\frac{2 \pi i}{\lambda f} \vec{x}_{1} \cdot \vec{x}_{1}^{\prime}\right) T\left(\vec{x}_{1}^{\prime}\right),
$$

with $\lambda$ being the wavelength. In arm 2 there is a single lens placed at a distance $z$ both from the source and from the detection plane 2; for simplicity we take the two lenses identical. 


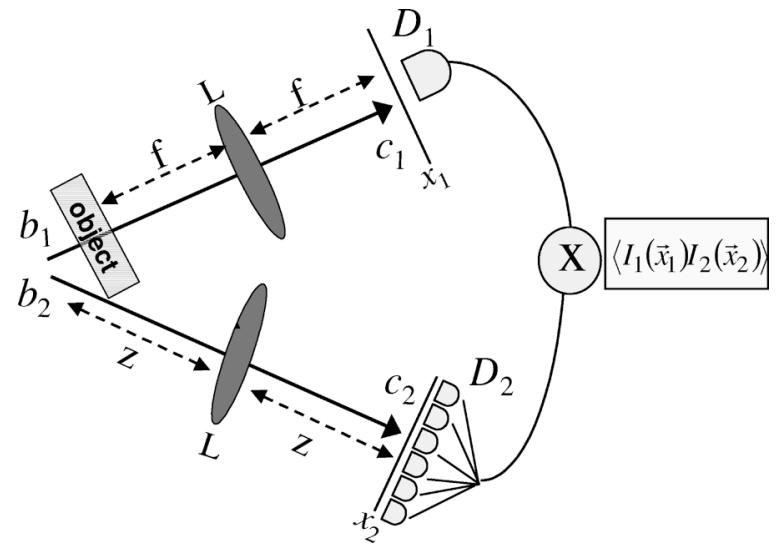

FIG. 2. Imaging scheme. $L$ denotes two identical lenses of focal length $f$. $D_{1}$ is a pointlike detector.The distance $z$ is either $z=f$ or $z=2 f$.

In the first example we assume $z=f$ so that $h_{2}\left(\vec{x}_{2}, \vec{x}_{2}^{\prime}\right)=$ $-(i / \lambda f) \exp \left[-(2 \pi i / \lambda f) \vec{x}_{2} \cdot \vec{x}_{2}^{\prime}\right]$. By inserting these propagators into Eq. (9) and taking into account Eq. (3), we obtain

$$
G_{\mathrm{th}}\left(\vec{x}_{1}, \vec{x}_{2}\right) \propto\left|\left\langle n\left(-\vec{x}_{2} \frac{2 \pi}{\lambda f}\right)\right\rangle_{\mathrm{th}} \tilde{T}\left(\left(\vec{x}_{2}-\vec{x}_{1}\right) \frac{2 \pi}{\lambda f}\right)\right|^{2},
$$

where $\widetilde{T}(\vec{q})=\int(d \vec{x} / 2 \pi) e^{-i \vec{q} \cdot \vec{x}} T(\vec{x})$ is the amplitude of the diffraction pattern from the object. This has to be compared with the result of the entangled case [see Eq. (7) of Ref. [7]],

$$
G_{\mathrm{PDC}}\left(\vec{x}_{1}, \vec{x}_{2}\right) \propto\left|U_{1}\left(-\vec{x}_{2} \frac{2 \pi}{\lambda f}\right) V_{2}\left(\vec{x}_{2} \frac{2 \pi}{\lambda f}\right) \widetilde{T}\left(\left(\vec{x}_{2}+\vec{x}_{1}\right) \frac{2 \pi}{\lambda f}\right)\right|^{2},
$$

where the combination $\vec{x}_{2}+\vec{x}_{1}$ appears instead of $\vec{x}_{2}-\vec{x}_{1}$ and $U_{1} V_{2}$ instead of $\langle n\rangle_{\mathrm{th}}$. In both the thermal and the PDC case the whole diffraction pattern from the object can be reconstructed via the correlation function. This holds provided that the spatial bandwidth $\Delta q$ is larger than the maximal transverse wave number $q$ in the diffraction pattern, or equivalently, provided that $l_{\text {coh }}<l_{0}$, where $l_{0}$ is the smallest scale of variation of the object spatial distribution. Thus, both cases have best performances of the scheme when spatially incoherent light $\left(l_{\text {coh }} \rightarrow 0\right)$ is used. In contrast, as it is well known, when $l_{\text {coh }}<l_{0}$ no information about the diffraction pattern of the object can be obtained without the correlations, i.e., if we detect the light intensity distribution in arm 1 with an array of pixels. In fact, one can easily obtain that

$$
\left\langle I_{1}\left(\vec{x}_{1}\right)\right\rangle=|r|^{2} \int \frac{d \widetilde{q}}{(\lambda f)^{2}}\left|\widetilde{T}\left(\vec{x}_{1} \frac{2 \pi}{\lambda f}-\vec{q}\right)\right|^{2}\langle n(\vec{q})\rangle_{\mathrm{th}} .
$$

For $l_{\text {coh }}<l_{\mathrm{o}},\langle n(\vec{q})\rangle_{\text {th }}$ can be taken out of the integral, and the resulting expression does not depend on $\vec{x}_{1}$ any more.

We incidentally remark that the result of Eq. (14) differs from what one would obtain with a standard HamburyBrown and Twiss scheme [15], where the object is placed in the thermal beam $a$ before the beam splitter. In that case, one would retrieve the Fourier transform of the modulus square of the object transmission function (see, e.g., Ref. [13]), thus losing any phase information. In our scheme instead, where the object is located in only one arm of the two, phase information about the object can be extracted and, e.g., the diffraction pattern from a pure phase object can be reconstructed.

In the second example, we set $z=2 f$, so that $h_{2}\left(\vec{x}_{2}, \vec{x}_{2}^{\prime}\right)$ $=\delta\left(\vec{x}_{2}+\vec{x}_{2}^{\prime}\right) \exp \left(-i\left|\vec{x}_{2}\right|^{2} \pi / \lambda f\right)$. Inserting this in Eq. (9) and taking into account (13), we get

$$
\begin{aligned}
G_{\mathrm{th}}\left(\vec{x}_{1}, \vec{x}_{2}\right) & \propto\left|\int d \vec{x}_{1}^{\prime} \Gamma\left(\vec{x}_{1}^{\prime}+\vec{x}_{2}\right) T^{*}\left(\vec{x}_{1}^{\prime}\right) e^{i(2 \pi / \lambda f) \vec{x}_{1}^{\prime} \cdot \vec{x}_{1}}\right|^{2} \\
& \left.\approx\left|\left\langle n\left(\vec{x}_{1} \frac{2 \pi}{\lambda f}\right)\right\rangle_{\mathrm{th}}\right|\right|^{2}\left|T\left(-\vec{x}_{2}\right)^{2}\right|,
\end{aligned}
$$

where in the second line $l_{\text {coh }}<l_{0}$ was assumed. Since the correlation function $\Gamma\left(\vec{x}_{1}^{\prime}+\vec{x}_{2}\right)=\left\langle a^{\dagger}\left(\vec{x}_{1}^{\prime}\right) a\left(-\vec{x}_{2}\right)\right\rangle$ is nonzero in a region of size $l_{\text {coh }}$ around $\vec{x}_{1}^{\prime}=-\vec{x}_{2}$, this condition ensures that $T\left(\vec{x}_{1}^{\prime}\right)$ is roughly constant in this region and it can be taken out from the convolution integral in Eq. (17), thus obtaining Eq. (18). In this example the intensity correlation function provides information about the image of the object. In the general case (17), the image reconstructed via the correlation function is a convolution of the object image with the second-order correlation function (3); therefore the thermal coherence length $l_{\text {coh }}$ fixes the resolution of the imaging scheme.

Under the same assumption $l_{\text {coh }}<l_{0}$, a similar result holds for the case of entangled beams [see Eq. (8) of Ref. [7]] [16]

$$
G_{\mathrm{PDC}}\left(\vec{x}_{1}, \vec{x}_{2}\right) \propto\left|T\left(-\vec{x}_{2}\right)\right|^{2}\left|U_{1}\left(\frac{2 \pi \vec{x}_{1}}{\lambda f}\right) V_{2}\left(-\frac{2 \pi \vec{x}_{1}}{\lambda f}\right)\right|^{2} .
$$

Also in this case the resolution of the scheme is limited by the finite transverse coherence length of the PDC beams.

This section shows that the classical correlation of the thermal beams offers imaging capabilities similar to those of the entangled PDC beams; both the image and the diffraction pattern of an object can be reconstructed and we can pass from one to the other by only operating on the optical setup in the reference arm. The performances of the imaging schemes based on classical correlation and on entanglement will be compared in Sec. V, both for what concerns the visibility, the statistical properties as well as the spatial resolution. The following section, instead, will explain the basic mechanism that allows correlated thermal imaging with such a degree of flexibility.

\section{NEAR- AND FAR-FIELD CORRELATION IN THE THERMAL AND ENTANGLED CASE}

As explained in detail in Ref. [7] the imaging schemes described in the preceding section have a peculiar feature. In the $z=f$ scheme the diffraction pattern reconstruction is made possible by the presence of spatial correlation in the far field of the correlated beams (momentum correlation of the photons). In the $z=2 f$ scheme, it is the presence of spatial cor- 
relation in the near-field (position correlation of the photons) that ensures the possibility of reconstructing the image. Our results for the thermal case may hence appear surprising if one has in mind the case of a coherent beam impinging on a beam splitter, where the two outgoing fields are uncorrelated, i.e., $G\left(\vec{x}_{1}, \vec{x}_{2}\right)=0$. However, when the input field is an intense thermal beam, i.e., the photon number per mode is not too small, the two outgoing beams are well correlated in space both in the near-field and in the far-field planes.

To prove this point, let us consider the number of photons detected in two small identical portions $S$ ("pixels") of the thermal beams in the near field immediately after the beam splitter, $N_{i}=\int_{S} d \vec{x} b_{i}^{\dagger}(\vec{x}) b_{i}(\vec{x}), i=1,2$, and the difference $N_{-}$ $=N_{1}-N_{2}$. Making use of the transformation (2), for $|r|^{2}$ $=|t|^{2}=1 / 2$ it can be proved that the variance $\left\langle\delta N_{-}^{2}\right\rangle=\left\langle N_{-}^{2}\right\rangle$ $-\left\langle N_{-}\right\rangle^{2}$ is given by

$$
\left\langle\delta N_{-}^{2}\right\rangle=\left\langle N_{1}\right\rangle+\left\langle N_{2}\right\rangle,
$$

which corresponds exactly to the shot noise level. Remarkably, Eq. (20) holds regardless of the statistical properties of the input beam $a$ provided that in the other input port there is the vacuum. On the other hand, by using the identity $\left\langle\delta N_{-}^{2}\right\rangle$ $=\left\langle\delta N_{1}^{2}\right\rangle+\left\langle\delta N_{2}^{2}\right\rangle-2\left\langle\delta N_{1} \delta N_{2}\right\rangle$ and taking into account that $\left\langle\delta N_{1}^{2}\right\rangle=\left\langle\delta N_{2}^{2}\right\rangle$ for $|r|^{2}=|t|^{2}$, the degree of spatial correlation is described by

$$
C=\frac{\left\langle\delta N_{1} \delta N_{2}\right\rangle}{\sqrt{\left\langle\delta N_{1}^{2}\right\rangle} \sqrt{\left\langle\delta N_{2}^{2}\right\rangle}}=1-\frac{\left\langle N_{1}\right\rangle}{\left\langle\delta N_{1}^{2}\right\rangle} .
$$

For any state $0 \leqslant|C| \leqslant 1$, where the upper bound is imposed by the Cauchy-Schwarz inequality. The lower bound corresponds to the coherent state, for which $\left\langle\delta N_{1}^{2}\right\rangle=\left\langle N_{1}\right\rangle$. For the thermal state, there is always some excess noise with respect to the coherent state $\left\langle\delta N_{1}^{2}\right\rangle>\left\langle N_{1}\right\rangle$, so that the correlation (21) never vanishes. Remarkably, a high degree of spatial correlation between beams $b_{1}$ and $b_{2}$ is ensured by the presence of a high level of excess noise in the input beam. As shown in detail in the Appendix, for thermal systems with a large number of photons, provided that the pixel size is on the order of $l_{\text {coh }}$ or larger, $\left\langle N_{1}\right\rangle /\left\langle\delta N_{1}^{2}\right\rangle \ll 1$, and $C$ can be made close to its maximum value.

Even more important, in the absence of losses it is not difficult to show that Eqs. (20) and (21) hold in any plane linked to the near-field plane by a Fresnel transformation. Let us assume that the propagation of beams $b_{1}, b_{2}$ is described by a linear and unitary kernel $H, b_{H, i}(\vec{x})$ $=\int d \vec{x}^{\prime} H\left(\vec{x}, \vec{x}^{\prime}\right) b_{i}\left(\vec{x}^{\prime}\right), i=1,2$. Then the form of the beamsplitter transformation (2) is preserved during propagation, provided that the thermal field $a$ is substituted by the propagated field $a_{H}(\vec{x})=\int d \vec{x}^{\prime} H\left(\vec{x}, \vec{x}^{\prime}\right) a\left(\vec{x}^{\prime}\right)$. Hence, Eqs. (20) and (21) also hold for $b_{H, 1}$ and $b_{H, 2}$, because these equations are just a consequence of the beam-splitter transformation (2) with $|r|^{2}=|t|^{2}=1 / 2$. Moreover, the field $a_{H}$ after propagation is still described by a thermal statistics, since the Gaussian statistics and the factorization property (4) of the fourthorder correlation function are preserved by a linear unitary transformation. However, the coherence properties of the field $a_{H}$ change upon propagation, as described by the well- known Van Cittert-Zernike theorem (see, e.g., Ref. [13]). In particular, in the far-field plane, where $H\left(\vec{x}, \vec{x}^{\prime}\right)=$ $-(i / \lambda f) \exp \left[-(2 \pi i / \lambda f) \vec{x} \cdot \vec{x}^{\prime}\right]$, they are described by a secondorder correlation function

$$
\left\langle a_{H}^{\dagger}(\vec{x}) a_{H}\left(\vec{x}^{\prime}\right)\right\rangle \propto \int d \vec{y} \int d \vec{y}^{\prime} e^{i(2 \pi / \lambda f)\left(\vec{x} \cdot \vec{y} \cdot \vec{x}^{\prime} \cdot \vec{y}^{\prime}\right)} \Gamma\left(\vec{y}, \vec{y}^{\prime}\right) .
$$

By assuming translational spatial invariance of the source, the far-field correlation function (22) is proportional to $\delta(\vec{x}$ $-\vec{x}^{\prime}$ ), as can be easily verified by substituting Eq. (3) into Eq. (22). However, when this unrealistic assumption is removed, the finite transverse size of the source $w_{S}$ has the effect that the correlation length of the function (22) is also finite and is inversely proportional to $w_{S}$, as shown by the Van CittertZernike theorem. Hence, $l_{\text {coh }}^{\prime} \propto \lambda f / w_{s}$, represents the coherence length in the far-field plane. We can thus conclude that a high level of pixel-by-pixel correlation can be observed also in the far-field plane, provided that the size of the detection regions $S$ is not too small with respect to $l_{\text {coh }}^{\prime}$, and the thermal beam is sufficiently intense (see the discussion in the Appendix).

We remark that despite $C$ can be made close to 1 by increasing the mean number of photons, it never reaches the quantum level, as shown by Eq. (20).

For the entangled beams produced by PDC, spatial correlation is present both in the near and in the far field, with the ideal result $\left\langle\delta N_{-}^{2}\right\rangle=0, C=1$ in both planes [11]. In this case, the far-field correlation is between symmetric pixels, and the coherence length in the far field is inversely proportional to the pump beam waist, which therefore in this context plays the same role as the source size $w_{S}$ for the thermal beams.

In Ref. [7] we analyzed the effect of replacing the pure PDC entangled state with two mixtures that exactly preserve the spatial signal-idler quantum correlations, either in the far or in the near field. It turned out that when considering the "far-field mixture" the pure state results could be exactly reproduced in the $z=f$ configuration of Fig. 2, but no information about the image was present in the $z=2 f$ configuration. The converse is true considering the "near-field mixture." This result is a consequence of the fact that the farfield intensity and the near-field intensity are noncommuting operators, and in the absence of quantum entanglement between the two beams they cannot simultaneously be correlated up to a perfect degree (this point is related to that raised in Ref. [17]). This led us to argue that only in the presence of quantum entanglement the whole set of results can be obtained by solely changing the setup in reference arm 2 [7].

However, nothing prevents two nonentangled beams to be correlated in both planes up to an imperfect degree. The two beams generated by splitting thermal light are actually imperfectly correlated both in the near and in the far field; but by using intense thermal light, the classical intensity correlation is strong enough to reproduce qualitatively the results of both the $z=f$ and the $z=2 f$ configuration. 


\section{IMAGING PERFORMANCES IN THE CLASSICAL AND QUANTUM REGIMES}

A complete comparison of the performances in the classical and quantum regimes requires extended numerical investigations describing realistic thermal sources, which are outside the scope of this paper.

However, some general remarks can be made concerning the key issue of the visibility of the information in the two regimes. The information about the object is retrieved by subtracting the background term $\left\langle I_{1}\left(\vec{x}_{1}\right)\right\rangle\left\langle I_{2}\left(\vec{x}_{2}\right)\right\rangle$ from the measured correlation function (6), as indicated in Eq. (7). A measure of this visibility is given by evaluating the following quantity in relevant positions:

$$
\mathcal{V}=\frac{G\left(\vec{x}_{1}, \vec{x}_{2}\right)}{\left\langle I_{1}\left(\vec{x}_{1}\right) I_{2}\left(\vec{x}_{2}\right)\right\rangle}=\frac{G\left(\vec{x}_{1}, \vec{x}_{2}\right)}{\left\langle I_{1}\left(\vec{x}_{1}\right)\right\rangle\left\langle I_{2}\left(\vec{x}_{2}\right)\right\rangle+G\left(\vec{x}_{1}, \vec{x}_{2}\right)}
$$

with $0 \leqslant \mathcal{V} \leqslant 1$

A first remark concerns the presence of $\langle n(\vec{q})\rangle_{\text {th }}$ in Eq. (9) in place of $U_{1}(\vec{q}) V_{2}(-q)$ in Eq. (11). As a consequence, in the thermal case $G_{\mathrm{th}}\left(\vec{x}_{1}, \vec{x}_{2}\right)$ scales as $\langle n(\vec{q})\rangle_{\mathrm{th}}^{2}$. In the entangled case, $G_{\mathrm{PDC}}\left(\vec{x}_{1}, \vec{x}_{2}\right) \quad$ scales as $\left|U_{1}(\vec{q}) V_{2}(-q)\right|^{2}=\langle n(\vec{q})\rangle_{\mathrm{PDC}}$ $+\langle n(\vec{q})\rangle_{\mathrm{PDC}}^{2}$, where $\langle n(\vec{q})\rangle_{\mathrm{PDC}}=\left|V_{2}(-\vec{q})\right|^{2}=\left|V_{1}(\vec{q})\right|^{2}$ is the mean number of photons per mode in the PDC beams, and $\left|U_{1}(\vec{q})\right|^{2}=1+\left|V_{1}(\vec{q})\right|^{2}$ (see, e.g., Ref. [11]). The difference between the two cases is immaterial when the mean photon number is large, while it emerges clearly in the small photon number regime $[\langle n(\vec{q})\rangle \ll 1]$. Actually, in the thermal case the visibility does not exceed the value $1 / 2$, whatever the value of $\langle n(\vec{q})\rangle_{\mathrm{th}}$, since $G_{\mathrm{th}}\left(\vec{x}_{1}, \vec{x}_{2}\right)$ scales in the same way as the background term. On the contrary, in the PDC case the visibility can approach the value 1 in the small photon number regime, since in this case the leading scale of $G_{\mathrm{PDC}}\left(\vec{x}_{1}, \vec{x}_{2}\right)$ is $\langle n(\vec{q})\rangle_{\mathrm{PDC}}$ and this term becomes dominant with respect to the background $\left\langle I_{1}\left(\vec{x}_{1}\right)\right\rangle\left\langle I_{2}\left(\vec{x}_{2}\right)\right\rangle \propto\langle n(\vec{q})\rangle_{\mathrm{PDC}}^{2}$. Hence, in the regime of single-photon pair detection the entangled case presents a much better visibility of the information with respect to classically correlated thermal beams (see also Ref. [18]).

A second remark concerns the role of the temporal argument. Standard calculations show that the visibility scales as the ratio between the coherence time of the source $\tau_{\text {coh }}$ and the detection time (see also Refs. [10,19]). This implies that conventional thermal sources, with very small coherence times, are not suitable for the schemes studied here. A suitable source should present a relatively long coherence time, as for example a sodium lamp, for which $\tau_{\text {coh }} \approx 10^{-10} \mathrm{~s}$ [15], or the chaotic light produced by scattering a laser beam through a random medium (see, e.g., Ref. [20]).

As a special example of a thermal source, one can consider the signal field or the idler field generated by PDC. Figure 3 shows the results of a numerical simulation [21] for the reconstruction of the diffraction pattern of a double slit, in the scheme $z=f$ of Fig. 2. It compares the use of the entangled signal and idler beams [curve $a$ ], and two classically correlated beams obtained by symmetrically splitting the signal beam [curve $b$ ]. The parametric gain is such that $\langle n(\vec{q})\rangle_{\mathrm{PDC}} \approx 750$ at its maximum for $a$, and $\langle n(\vec{q})\rangle_{\mathrm{PDC}}$ $\approx 1500$ for $b$, so that the mean photon numbers of beams $b_{1}$,

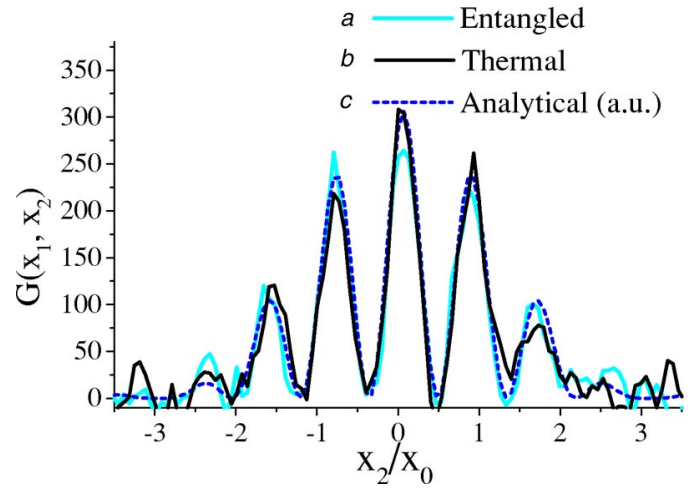

FIG. 3. Numerical simulation of the reconstruction of the diffraction pattern of a double slit in the scheme $z=f$ of Fig. 2 . $G\left(\vec{x}_{1}, \vec{x}_{2}\right)$ is plotted vs $\vec{x}_{2}$ after $10^{4}$ pump shots for $a$ entangled signal/idler beams from PDC, $b$ classically correlated beams by splitting the signal beam. $c$ is the analytical result of Eq. (14). Parameters are those of a $4 \mathrm{~mm} \beta$-barium-borate crystal ( $=16.6 \mu \mathrm{m}, \tau_{\mathrm{coh}}=0.97 \mathrm{ps}$ ). The pump waist is $664 \mu \mathrm{m}$, and the pulse duration is $1.5 \mathrm{ps}$. The slits are $36 \mu \mathrm{m}$ wide and slit separation is $122 \mu \mathrm{m} . x_{0}$ is defined as $\Delta q \lambda f /(2 \pi)$.

$b_{2}$ are approximately equal in the two simulations. From our simulations it clearly emerged that the number of pump shots necessary for reconstructing the diffraction pattern up to a desired accuracy is the same for both curves $a$ and $b$. Notice that Fig. 3 plots only $G\left(\vec{x}_{1}, \vec{x}_{2}\right)$, which contains the object information and represents the relevant part of the intensity correlation. This was obtained by subtracting a large background term $\left\langle I_{1}\left(\vec{x}_{1}\right)\right\rangle\left\langle I_{2}\left(\vec{x}_{2}\right)\right\rangle$ from the correlation function of the intensities $\left\langle I_{1}\left(\vec{x}_{1}\right) I_{2}\left(\vec{x}_{2}\right)\right\rangle$. Consideration of these quantities (not shown in the figure) allowed us to calculate the visibility $\mathcal{V}$, as defined by Eq. (23), which turned out to be $\mathcal{V} \approx 0.05$ in both cases. Although this is a rather poor visibility, the crucial point is that the fringes shown in Fig. 3 could be correctly reconstructed after a reasonable number of pump shots.

The simulations of Fig. 3 were repeated but changing the setup in the reference arm to the $z=2 f$ configuration. The results are shown in Fig. 4, and confirm that the classical correlation can be used to reconstruct both the diffraction pattern and the image of the object by operating only in the optical setup in the reference arm 2. Also in this case the efficiency of the reconstruction is the same for curves $a$ and $b$.

These examples clearly show that in the regime of high photon number the quantum and classical correlations offer similar performances.

Another important aspect that emerges from these examples is that the classical and quantum-imaging schemes apparently offer the same spatial resolution. This is most evident in the plot of Fig. 4, where the spatial resolution of both schemes is not good enough to reproduce the sharp details of the double slit image, but the reconstructed images are almost identical. As a matter of fact the analytical results-given by the general formulas (9)-(11) for the correlation function of intensity fluctuations, the results (14) and (15) for the diffraction pattern reconstruction, and the results (18) and (19) for the image reconstruction-show clearly 


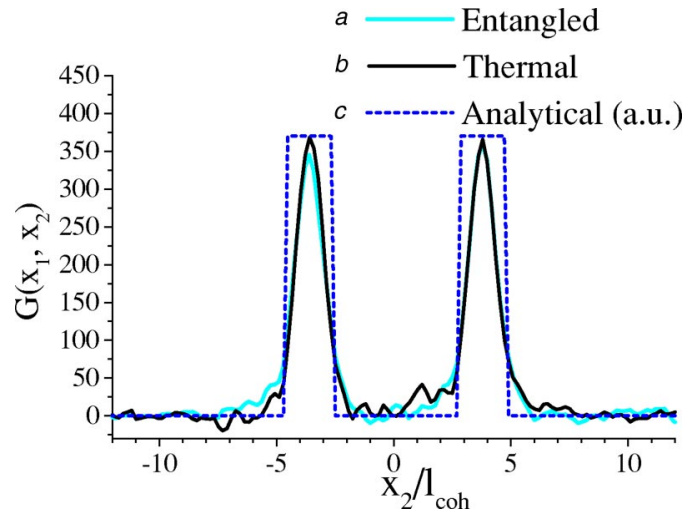

FIG. 4. Numerical simulation of the reconstruction of the image of a double slit in the scheme $z=2 f$ of Fig. 2. $G\left(\vec{x}_{1}, \vec{x}_{2}\right)$ is plotted vs $\vec{x}_{2}$ after $10^{4}$ shots for $a$ entangled signal and idler beams from PDC, $b$ classically correlated beams by splitting the idler beam. $c$ is the analytical result of Eq. (18). Parameters are as in Fig. 3.

that the spatial resolution of both schemes depends only on the spatial coherence properties of the sources. Provided that the spatial coherence properties of the classical source emulate those of the entangled source, there is no reason why the two schemes should offer different spatial resolutions. This requirement is not unrealistic at all, since the typical transverse coherence length of the entangled beams from PDC is on the order of tens of microns. An example of "thermal" light whose coherence properties can be engineered is offered by, e.g., chaotic radiation obtained by scattering laser light through random media [20].

A final remark concerns the form of the analytical results of Eqs. (14) and (15) for the diffraction pattern reconstruction. Specifically, the diffraction pattern on the rhs of Eq. (15) depends on the sum $\vec{x}_{1}+\vec{x}_{2}$ of the positions of the pixels in the detection planes of beam 1 and 2. This feature was exploited in the experiment of Ref. [22] (see also Ref. [23]), which was performed by registering coincidence counts of pairs of photons generated by PDC in a configuration similar to the $z=f$ scheme of Fig. 2 [24]. The interference fringes from a double slit were observed by scanning the pixel detectors in the two beams together, i.e., with $\vec{x}_{1}=\vec{x}_{2}$. A halving of the period of the interference fringes with respect to those observed by illuminating the object with coherent light was then observed. This effect was claimed to be a consequence of the entanglement of the two-photon state. This effect is evident from Eq. (15), where by setting $\vec{x}_{1}+\vec{x}_{2}=\vec{x}$ the PDC correlation function gives $|\widetilde{T}[2 \vec{x}(2 \pi / \lambda f)]|^{2}$. By inspecting Eq. (14), we notice that the same effect could in principle be observed in the scheme that uses the classically correlated thermal beams, provided that the pixels in the detection planes of the two beams are scanned symmetrically, i.e., setting $\vec{x}_{1}=-\vec{x}_{2}$ [25]. Therefore, the discussion is open whether quantum entanglement has a crucial role in this observed halving of the period of the interference fringes, or whether this is just a consequence of the spatial correlation of the two beams and of the particular detection scheme used.

\section{CONCLUSIONS}

In conclusion we have suggested a way of producing classically correlated beams suitable for correlated imaging.
These beams are the outcome of mixing an intense thermal beam with the vacuum state on a beam splitter. We have shown a deep analogy between the use of entangled beams originating from PDC and these classically correlated beams. The analogy arises because of the similar structure of Eqs. (9) and (11), which implies that the outcomes of correlation measurements with the classical source can emulate those obtained with the entangled beams, for any choice of the test and reference arm optical setups. This holds provided the spatial coherence properties of the source are chosen to mimic the marginal statistics of the individual PDC beams.

This analogy relies on the high level of spatial correlation that exists between the two beams emerging from the beam splitter as a consequence of the large excess noise of the thermal input. A key point is that a pixel by pixel correlation is present not only in the near-field plane immediately after the beam splitter but also in the far-field plane. Although the correlation is limited by shot noise, it allows to reconstruct both the image and the diffraction pattern of an object by only acting on the optical setup of the reference arm. This was hitherto thought of as a feature that truly required entanglement between the two beams [7].

We have investigated the imaging performances of the quantum and classical schemes. Both our analytical results and a specific numerical example show that the spatial resolution limitations of the two schemes have similar origins, namely, the finite transverse coherence length of the light. Thus, also in this respect the classical beams mimic the results of the entangled beams.

On the other hand, in the small photon number regime a definite advantage of the quantum configuration is represented by a better visibility, as it was already recognized in other contexts (see, e.g., Ref. [26]). Thus, in imaging schemes where the visibility represents a crucial issue one should take this into account. However, as the number of photons per mode becomes large this advantage disappears and the visibility tends to be the same for the quantum and the classical source. This result suggests that the peculiar difference between the use of the two kind of sources is not given by the entanglement, but rather by the possibility of working in the photon counting regime in the quantum case.

A further advantage of the PDC source may lie in the possibility of using a fraction of the source laser as a reference field in order to perform balanced homodyne detection. We will show in a future theoretical work that the homodyne scheme makes it possible to perform phase-sensitive correlated imaging with a high degree of visibility even in the large photon number regime [27]. Another advantage of using the PDC source relies on the possibility of multiwavelength imaging, as mentioned in the introduction.

Our results implies that it is possible to perform coherent imaging without spatial coherence by using thermal light in combination with a beam splitter. This is reminiscent of the Hanbury-Brown and Twiss interferometric method for determining the stellar diameter [13], as well as of detecting the fringes arising from interference of two independent thermal sources [28]. However, here we define a technique to achieve a fully coherent imaging of the object through correlation measurements with a high degree of flexibility. Moreover, since the required correlation is classical, a high quantum efficiency of the detectors is not necessary. 


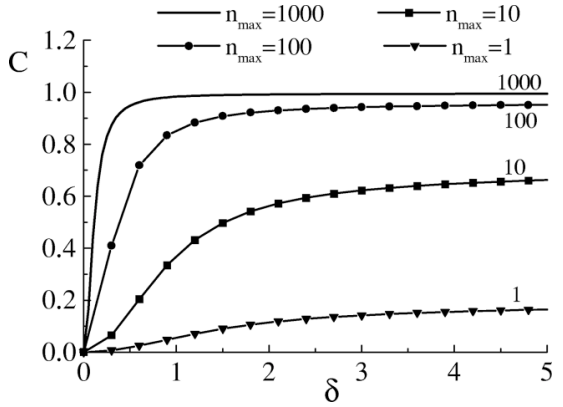

FIG. 5. Degree of spatial correlation $C$ between two identical detection regions of the beams obtained by splitting thermal light, as a function of the ratio $\delta$ between the pixel size and the coherence length. $n_{\max }$ is the mean photon number in the most intense mode. $C=1$ represents the maximum degree of correlation.

The results of this paper suggest experiments of correlated imaging with thermal light, which could provide a useful test bed for the imaging experiments with quantum entangled sources. Experiments like that simulated by the numerics of Sec. V may open possibilities, offered by the combination of the correlated imaging from entangled beams and from classically correlated beams.

\section{ACKNOWLEDGMENTS}

We are grateful to Alexander Sergienko, Bob Boyd, Ryan Bennink, and Eric Lantz for stimulating discussions. This work was carried out within the framework of the FET project QUANTIM of the EU, of the PRIN project of MIUR "Theoretical Study of Novel Devices Based on Quantum Entanglement," and of the INTAS project "Non-classical Light in Quantum Imaging and Continuous Variable Quantum Channels." M.B. acknowledges financial support from the Danish Technical Research Council (STVF).

\section{APPENDIX}

This appendix investigates how the degree of spatial correlation between the beams obtained by splitting thermal light depends on the size of the pixels used to detect the light, and on the mean number of thermal photons.

As shown by Eq. (21) a high level of spatial correlation can be present between the beams $b_{1}, b_{2}$ after the beam splitter as a consequence of a high level of excess noise in the thermal beam $a$. The relevant quantity to consider is the ratio $\left\langle: \delta N_{1}^{2}:\right\rangle /\left\langle N_{1}\right\rangle$, where $\left\langle: \delta N_{1}^{2}:\right\rangle$ is defined by $\left\langle\delta N_{1}^{2}\right\rangle=\left\langle N_{1}\right\rangle$ $+\left\langle: \delta N_{1}^{2}:\right\rangle$, and represents the noise in excess with respect to the coherent state level.

By using the beam-splitter transformation (2) and the factorization property (4) of the fourth-order thermal correlation function, we get

$$
\left\langle: \delta N_{1}^{2}:\right\rangle=|r|^{4} \int_{S} d \vec{x} \int_{S} d \vec{x}^{\prime}\left|\Gamma\left(\vec{x}-\vec{x}^{\prime}\right)\right|^{2},
$$

where $\Gamma\left(\vec{x}-\vec{x}^{\prime}\right)$ is the thermal correlation function defined by Eq. (3). On the other hand, the mean number of photons

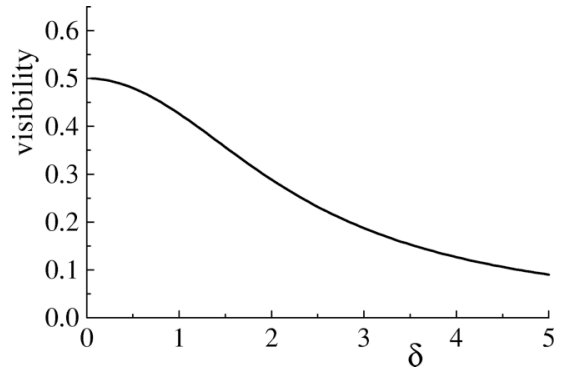

FIG. 6. Visibility $\mathcal{V}_{S}$ of the spatial correlation between two identical detection regions of the beams obtained by splitting thermal light, as a function of the ratio $\delta$ between the pixel size and the coherence length.

detected over the pixel is

$$
\left\langle N_{1}\right\rangle=|r|^{2} S \Gamma(0) \propto|r|^{2} \frac{S}{l_{\text {coh }}^{2}} n_{\max },
$$

where we introduced the parameter

$$
n_{\max }=\langle n(\vec{q}=0)\rangle_{\mathrm{th}}
$$

that represents the mean number of photon in the most intense mode $\vec{q}=0$ in the spectrum. Moreover, we used $\Gamma(0)$ $=\int d \vec{q} /(2 \pi)^{2}\langle n(\vec{q})\rangle_{\mathrm{th}} \propto 1 / l_{\mathrm{coh}}^{2} n_{\max }$, where the proportionality constant depends on the actual shape of the spectrum. When the pixel size is much smaller than $l_{\text {coh }}$, the correlation function in Eq. (A1) is approximately constant over the integration regions $S$, so that

$$
\left\langle: \delta N_{1}^{2}:\right\rangle \rightarrow|r|^{4} S^{2} \Gamma(0)^{2}=\left\langle N_{1}\right\rangle^{2} .
$$

Hence, in this limit of a small detection region the noise takes the form of the usual single-mode result for thermal light $\left\langle\delta N_{1}^{2}\right\rangle=\left\langle N_{1}\right\rangle+\left\langle N_{1}\right\rangle^{2}$. However, as shown by Eq. (A2) the mean number of photons is also small in this limit, so that the excess noise and the correlation tend to be both small.

On the other side, when the detection regions grow much larger than the coherence area the excess noise does not scale any more with the square of the mean number of detected photons. In the limit of large detection regions the r.h.s. of Eq. (A1) can be approximated as

$$
\begin{aligned}
\left\langle: \delta N_{1}^{2}:\right\rangle & \rightarrow|r|^{4} S \int_{\text {trv.plane }} d \vec{\xi}|\Gamma(\vec{\xi})|^{2} \\
& =|r|^{4} S \int \frac{d \vec{q}}{(2 \pi)^{2}}\langle n(\vec{q})\rangle_{\text {th }}^{2} \\
& \propto|r|^{4} \frac{S}{l_{\text {coh }}^{2}} n_{\text {max }}^{2} \approx|r|^{2} n_{\max }\left\langle N_{1}\right\rangle .
\end{aligned}
$$

For a large detection region the excess-noise scales proportionally to the mean number of detected photons, so that the ratio $\left\langle: \delta N_{1}^{2}:\right\rangle /\left\langle N_{1}\right\rangle$ reaches a limiting value, which depends on $n_{\max }$.

We have investigated more in detail the role of the size of the detection region in the degree of correlation by assuming 
square pixels with sides of size $\Delta$, as well as a Gaussian

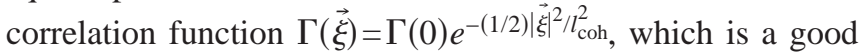
approximation of a smooth correlation function decaying on a distance $l_{c o h}$. By substituting this in Eq. (A1) and using the result of Eq. (21), we get the two relevant asymptotic behaviors of the degree of correlation (21):

$$
\begin{gathered}
C \rightarrow \frac{1}{4 \pi} \frac{\delta^{2} n_{\max }}{1+\frac{1}{4 \pi} \delta^{2} n_{\max }} \text { for } \delta \ll 1, \\
C \rightarrow \frac{1}{4} \frac{n_{\max }}{1+\frac{1}{4} n_{\max }} \text { for } \delta \rightarrow \infty,
\end{gathered}
$$

where $\delta=\Delta / l_{\text {coh }}$ is the ratio of the pixel size to the coherence length. The curves in Fig. 5 show the general behavior of the degree of correlation $C$ as a function of the pixel size for different values of the mean photon number per mode $n_{\max }$. The consequence of this figure is that when the input thermal beam is intense enough, a high degree of spatial correlation can be achieved. This is provided that the pixel size is not too small with respect to the coherence length describing the decay of the thermal spatial correlation function.
Another relevant parameter that characterizes the spatial correlation between two beams is the visibility of the correlation, which in the spirit of Eq. (21) we define here as

$$
\mathcal{V}_{S}=\frac{\left\langle\delta N_{1} \delta N_{2}\right\rangle}{\left\langle N_{1} N_{2}\right\rangle} .
$$

This definition is analogous to that of Eq. (23), where, however, a small pixel was implicitly considered so that no integration over the pixel area was performed. This quantity is easily calculated in the same manner as above. Figure 6 plots the visibility of the spatial correlation between the thermal beams as a function of the pixel size scaled to the coherence length. This plot was obtained under the same assumption of Fig. 5. The visibility turns out not to depend on the mean thermal photon number $n_{\max }$, so that only one curve is plotted.

Unlike the degree of correlation, the best visibility is obtained when the detection pixel is not large with respect to the coherence length. The main conclusion of this appendix is therefore: in order to achieve both a good visibility as well as a high degree of spatial correlation, the best choice is a detection pixel with a size approximately equal to the coherence area.
[1] A. V. Belinskii and D. N. Klyshko, JETP 78, 259 (1994) [Zh. Eksp. Teor. Fiz. 105, 487 (1994).

[2] D. V. Strekalov, A. V. Sergienko, D. N. Klyshko, and Y. H. Shih, Phys. Rev. Lett. 74, 3600 (1995).

[3] T. B. Pittman, Y. H. Shih, D. V. Strekalov, and A. V. Sergienko, Phys. Rev. A 52, R3429 (1995).

[4] P. H.S. Ribeiro, S. Padua, J. C. Machado da Silva, and G. A. Barbosa, Phys. Rev. A 49, 4176 (1994).

[5] A. F. Abouraddy, B. E.A. Saleh, A. V. Sergienko, and M. C. Teich, Phys. Rev. Lett. 87, 123602 (2001).

[6] A. F. Abouraddy, B. E.A. Saleh, A. V. Sergienko, and M. C. Teich, J. Opt. Soc. Am. B 19, 1174 (2002).

[7] A. Gatti, E. Brambilla, and L. A. Lugiato, Phys. Rev. Lett. 90, 133603 (2003).

[8] R. S. Bennink, S. J. Bentley, and R. W. Boyd, Phys. Rev. Lett. 89, 113601 (2002).

[9] M. D'Angelo and Y. H. Shih, e-print quant-ph/0302146.

[10] B. E. A Saleh, A. F. Abouraddy, A. V. Sergienko, and M. C. Teich, Phys. Rev. A 62, 043816 (2000).

[11] E. Brambilla, A. Gatti, M. Bache, and L. A. Lugiato, Phys. Rev. A 69023802 (2004).

[12] M. I. Kolobov, Rev. Mod. Phys. 71, 1539 (1999).

[13] L. Mandel and E. Wolf, Optical Coherence and Quantum Optics (Cambridge University Press, Cambridge, 1995).

[14] O. Svelto, Principles of Lasers (Plenum Press, New York, 1982).

[15] R. Hanbury-Brown and R. Q. Twiss, Nature (London) 177, 27 (1956).

[16] There is unfortunately a misprint in Eq. (8) of Ref. [7], so that the argument of the function $T$ is $\vec{x}_{2}$ instead of $-\vec{x}_{2}$.

[17] J. C. Howell, R. S. Bennink, S. J. Bentley, and R. W. Boyd, e-print quant-ph/0309122.
[18] A. V. Belinsky and D. N. Klyshko, Phys. Lett. A 166, 303 (1992).

[19] A. Gatti, E. Brambilla, and L. A. Lugiato, Proc. SPIE 5161, 192 (2004).

[20] W. Martiessen and E. Spiller, Am. J. Phys. 32, 919 (1964).

[21] In our numerical simulations we dropped the translational invariance in both space and time of the input beam, and thus we also took into account the temporal variable (which was ignored in the analytical treatment). The simulations were done in $2 D+1$ dimensions, i.e. including one transverse dimension (along the walk-off direction) as well as the time dimension, and propagating along the $z$-direction of the crystal. The calculated intensities were integrated over time since the pulse length was much shorter than the response time of any available detectors with spatial resolution. See [11] for more details.

[22] M. D'Angelo, M. V. Chekhova, and Y. Shih, Phys. Rev. Lett. 87, 013602 (2001).

[23] R. Shimizu, K. Edamatsu, and T. Itoh, Phys. Rev. A 67, 041805 (2003).

[24] The scheme of the experiments of Ref. [20] is slightly different from the $z=f$ scheme of Fig. 2, and corresponds to having an object in both arms. However, this only has the effect that the Fourier transform of the square of the object transmission function is observed instead of the Fourier transform of the object transmission function, which is irrelevant when the object is a double slit.

[25] One can easily imagine a setup where in arm 1 there is an additional optical element that flips the beam transverse distribution, so that $\vec{x}_{1}$ can be scanned together with $\vec{x}_{2}$ as in the experiments of [20] and [21].

[26] Z. Y. Ou and L. Mandel, J. Opt. Soc. Am. B 7, 2127 (1990).

[27] M. Bache, E. Brambilla, A. Gatti, and L. A. Lugiato, Phys. 
Rev. A (to be published), e-print quant-ph/0402160.

[28] S. J. Ku, D. T. Smithey, and M. G. Raymer, Phys. Rev. A 43, 4083 (1991).
[29] A. Gatti, E. Brambilla, M. Bache, and L. A. Lugiato, Phys. Rev. Lett. (to be published), e-print quant-ph/0307187. 